\title{
AMPK/SIRT1/p38 MAPK signaling pathway regulates alcohol-induced neurodegeneration by resveratrol
}

\author{
XINYI GU ${ }^{1 *}$, ZHENGXU CAI $^{2 *}$, MING CAI $^{1}, \mathrm{KUN} \mathrm{LIU}^{1}, \mathrm{DAN} \mathrm{LIU}^{1}$, \\ QINSONG ZHANG ${ }^{1}$, JING TAN ${ }^{1}$ and QIANG MA ${ }^{1}$ \\ ${ }^{1}$ Department of Neurology, The Affiliated Zhongshan Hospital of Dalian University; ${ }^{2}$ Department of Neurology, \\ The First Affiliated Hospital of Dalian Medical University, Dalian, Liaoning 116001, P.R. China
}

Received October 17, 2016; Accepted June 8, 2017

DOI: $10.3892 / \mathrm{mmr} .2018 .8482$

\begin{abstract}
Resveratrol has also been approved for use in enhancing plant disease resistance and reducing pesticide use. A number of studies have shown that the disease resistance of crops treated with resveratrol is markedly improved. The aim of the present study was to examine the protective effect of resveratrol against alcohol-induced neurodegeneration occurred and its association with AMP-activated protein kinase (AMPK)/sirtuin 1 (SIRT1)/p38 in rats and humans. ELISA, caspase-3 activity and western blot analyses were employed in the present study. Sprague-Dawley rats and human neuroblastoma SH-SY5Y cells were treated with alcohol to establish the alcohol-induced model. Resveratrol protected against alcohol-induced neuron damage in the hippocampus of the rats. Treatment with resveratrol also inhibited the alcohol-induced inflammatory response, oxidative stress, caspase-3 activities and B-cell lymphoma (Bcl-2)-associated X protein/Bcl-2 in the alcohol-induced rat. Resveratrol also reduced the upregulated protein expression of AMPK and SIRT1, preventing the pro-apoptotic alcohol-induced protein expression of p38 in the rats exposed to alcohol. The downregulation of AMPK suppressed the expression of SIRT1 and activated the expression of p38 in the SH-SY5Y cell model. Taken together, the data obtained suggested that resveratrol protected against alcohol-induced neurodegeneration via the AMPK/SIRT1/p38 pathway in rats and humans.
\end{abstract}

Correspondence to: Dr Qiang Ma, Department of Neurology, The Affiliated Zhongshan Hospital of Dalian University, 6 Jiefang Street, Dalian, Liaoning 116001, P.R. China

E-mail: qqiangmama@163.com

"Contributed equally

Key words: resveratrol, alcohol, neurodegeneration, AMP-activated protein kinase, sirtuin $1, \mathrm{p} 38$

\section{Introduction}

In the history of world civilization and with the evolution of wine culture, wine has become a common pharmacologically active beverage in society, and is consumed for social and recreational purposes. However, the consumption alcohol also increases the risk of health problems, and particularly affects future generations. In the late 1960s and early 1970s, clinical studies in the United States and France showed that drinking alcohol during pregnancy can lead to a variety of abnormalities, namely fetal alcohol syndrome (1). Fetal alcohol syndrome refers to the birth defects resulting from alcohol consumption by both parents, particularly when the mother consumes alcohol prior to or during pregnancy (2). A number of studies have confirmed that exposure to alcohol during pregnancy affects fetal growth and development, predominantly in various organ deformities of the central nervous system $(2,3)$.

The mechanism underlying the neurotoxicology of alcohol remains to be fully elucidated. It has been suggested that alcohol may have an effect by inhibiting the N-methyl-D-aspartate receptor (NMDA) receptor and activating GABA receptors, and can promote apoptosis by affecting the activities of $\mathrm{Na}^{+} / \mathrm{K}^{+}$-ATP enzymes, the adenosine acid cyclization enzyme, and opening of $\mathrm{Ca}^{2+}$ channels. Others have suggested that the pharmacological and toxicological mechanisms of alcohol may work through ceramide (4,5). As an important cellular signal transduction carrier, ceramide is involved in a variety of cellular signal transduction pathways, and has multiple cellular physiological functions, including regulation of the cell cycle, cell differentiation and proliferation, regulation of apoptosis, and being involved in stress, immunity and inflammation $(6,7)$.

The quantity of resveratrol produced by plants is associated with the stress experienced by the plants. The function universality of resveratrol lies in the diversity of the targets, of which the acting sites may include membrane and intracellular receptors, signal molecules and various enzymes, the oxidation system, DNA repair systems and transcription factors, cell proliferation, the cell cycle, differentiation and cell death (8). The signal transduction function of cells converts stimuli into signals, and the process usually consists of a series of biochemical reactions. Resveratrol can activate or inhibit a series of signal transduction pathways (9). In vitro experiments, in which resveratrol regulates the activity of 
sirtuin 1 (SIRT1), are difficult to replicate completely in vivo, therefore, resveratrol may act on SIRT1 through an indirect mechanism. Resveratrol can regulate glucose homeostasis in mammals by the deacetylation mediated by SIRT1 (9). The aim of the present study was to examine whether the protective effect of resveratrol protects against alcohol-induced neurodegeneration in rats and humans. In addition, the present study aimed to examine the association among the AMP-activated protein kinase (AMPK)/SIRT1/p38 MAPK signaling pathway and the protective effect of resveratrol on alcohol-induced neurodegeneration.

\section{Materials and methods}

Animals. All animal experiments were approved by the Institute Animal Care and Use Committee of Dalian University and were performed humanely. Male Sprague-Dawley rats (8-10-weeks-old, weighing 250-300 g, n=28) were housed at $22-24^{\circ} \mathrm{C}$ and $55-60 \%$ humidity under a $12 \mathrm{~h}$ light/dark cycle with free access to food and water. Rats were randomly assigned into a control group $(n=8)$, alcohol-induced group $(n=10)$ and resveratrol-treated group $(n=10)$. A total of 20 Sprague-Dawley rats from the alcohol-induced group and resveratrol-treated group were administered orally with $5 \mathrm{~g} / \mathrm{kg}$ ethanol $(25 \% \mathrm{v} / \mathrm{v})$ once a day for 10 days. The eight Sprague-Dawley rats in the control group were administered orally with $200 \mu \mathrm{l}$ normal saline. The 20 Sprague-Dawley rats in the resveratrol-treated group were administered orally with $30 \mathrm{mg} / \mathrm{kg}$ of resveratrol for 4 days.

Brain tissue collection. Rat was anesthetized with $35 \mathrm{mg} / \mathrm{kg}$ of pentobarbital sodium and sacrificed using decollation. Whole brain samples were obtained and sectioned to $50 \mathrm{~mm}$ thickness. Rat brain samples from rats in every group were fixed in $10 \%$ formalin buffer overnight at $4{ }^{\circ} \mathrm{C}$ and then dehydrated using 90\% ethanol for 30-60 min and 100\% ethanol for $2 \mathrm{~h}$. The tissues were cleared with xylene for $2 \mathrm{~h}$ and embedded in paraffin at $60^{\circ} \mathrm{C}$.

Cell culture. The human neuroblastoma SH-SY5Y cells were purchased from the Cell Bank of Type Culture Collection of the Chinese Academy of Sciences (Shanghai, China) and grown in Dulbecco's modified Eagle's medium (Gibco; Thermo Fisher Scientific, Inc., Waltham, MA, USA) supplemented with $10 \%$ fetal bovine serum (Gibco; Thermo Fisher Scientific, Inc.) at $37^{\circ} \mathrm{C}$ under $5 \% \mathrm{CO}_{2}$. The SH-SY5Y cells were exposed to $100 \mathrm{mM}$ of ethanol (Sigma-Aldrich; Merck KGaA, Darmstadt, Germany) for $24 \mathrm{~h}$ at $37^{\circ} \mathrm{C}$ in the alcohol-induced group and resveratrol-treated group. The SH-SY5Y cells in the resveratrol-treated group were exposed to $50 \mathrm{mg} / \mathrm{ml} \mathrm{resveratrol} \mathrm{for}$ 1 day at $37^{\circ} \mathrm{C}$.

ELISA. Blood samples were collected from rats in every group into a serum separator tube and centrifuged at $1,000 \mathrm{x} \mathrm{g}$ for $15 \mathrm{~min}$ at room temperature. Commercial ELISA kits (Nanjing Jiancheng Bioengineering Institute, Jiangsu, China) were used to analyze the levels of nuclear factor (NF)- $\kappa \mathrm{B}(\mathrm{H} 202)$, tumor necrosis factor (TNF)- $\alpha$ (H052), superoxide dismutase (SOD, A001-3) and glutathione (GSH, A006-2) levels, according to the manufacturer's protocols, at $450 \mathrm{~nm}$ absorbance using a

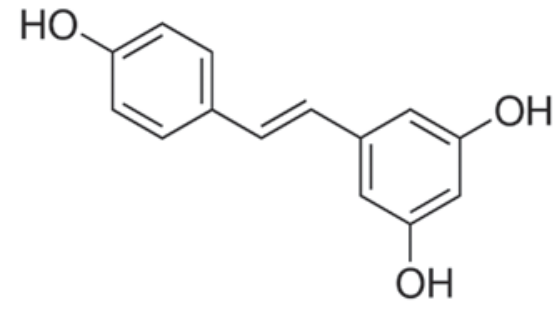

Figure 1. Chemical structure of resveratrol.

microplate reader (Bio-Rad Laboratories, Inc., Hercules, CA, USA).

Caspase-3 activity assay. The activity of caspase-3 was measured using a caspase-3 activity assay kit (Sangon Biotech Co., Ltd., Shanghai, China). The protein concentration was determined using a Bicinchoninic Acid kit (Beyotime Institute of Biotechnology). The proteins $(50 \mu \mathrm{g})$ were incubated with solution buffer at room temperature for $1 \mathrm{~h}$ and then incubated with $100 \mu \mathrm{l}$ Asp-Glu-Val-Asp-p-nitroaniline at $37^{\circ} \mathrm{C}$ for $6 \mathrm{~h}$. The activity of caspase- 3 was measured using a microplate reader (Bio-Rad Laboratories, Inc.) at an absorbance of $405 \mathrm{~nm}$.

Western blot analysis. The rat brain slices from rats in every group were homogenized in $100 \mu 1$ tissue lysis buffer (Beyotime Institute of Biotechnology) with protease inhibitor cocktail (Sigma-Aldrich; Merck KGaA) on ice for $30 \mathrm{~min}$ and centrifuged at $12,000 \mathrm{x}$ g for $10 \mathrm{~min}$ at $4{ }^{\circ} \mathrm{C}$. The protein concentration was determined using a Bicinchoninic Acid kit (Beyotime Institute of Biotechnology). The proteins $(50 \mu \mathrm{g})$ was separated by sodium dodecyl sulphate polyacrylamide gel electrophoresis (10-12\%) and transferred onto polyvinylidene fluoride membranes (Bio-Rad Laboratories, Inc.). The membranes were blocked with $5 \%(\mathrm{w} / \mathrm{v})$ non-fat dry milk in Tris-buffered saline with $0.1 \%$ Tween 20 for $2 \mathrm{~h}$ at room temperature and incubated with the following primary antibodies: B-cell lymphoma 2 (Bcl-2; sc-783, 1:1,000; Santa Cruz Biotechnology, Inc., Dallas, TX, USA) Bcl-2-associated X protein (Bax; sc-6236, 1:2,000; Santa Cruz Biotechnology,Inc.), AMPK (sc-25792, 1:1,500, Santa Cruz Biotechnology, Inc.), SIRT1 (sc-15404, 1:2,000; Santa Cruz Biotechnology, Inc.), p-p38 (sc-17852-R, 1:2,000; Santa Cruz Biotechnology, Inc.) and $\beta$-actin (sc-7210, 1:5,000; Santa Cruz Biotechnology, Inc.) at $4^{\circ} \mathrm{C}$ overnight. The membranes were then blocked with secondary antibodies (D110058, 1:20,000; Sangon Biotech Co., Ltd.) for $2 \mathrm{~h}$ at room temperature and developed with an enhanced chemiluminescence substrate solution (Applygen Technologies, Inc., Beijing, China) and analyzed using Image-ProPlus 6.0 software (Media Cybernetics, Inc., Rockville, MD, USA)

Small interfering RNA (siRNA) knockdown of AMPK. The SH-SY5Y cells $\left(3 \times 10^{5} /\right.$ well) in 12-well plates were incubated with Lipofectin (Gibco; Thermo Fisher Scientific, Inc.) with 200 nM AMPK-specific siRNA (sc-270395, Santa Cruz Biotechnology, Inc.) or control siRNA (sc-270395, Santa Cruz Biotechnology, Inc.) for $48 \mathrm{~h}$ at $37^{\circ} \mathrm{C}$. The effects of siRNA transfection was measured by western blot analysis as aforementioned. 


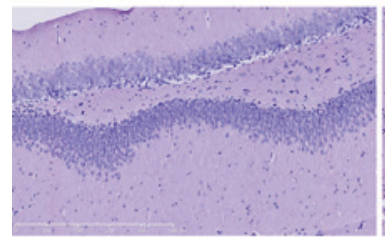

Control

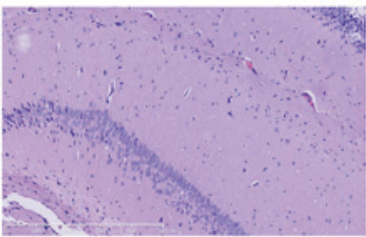

Alcohol

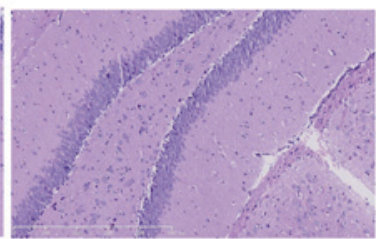

Resveratrol

Figure 2. Resveratrol protects against alcohol-induced neurodegeneration. Number of neuron cells (magnification, x10).

A

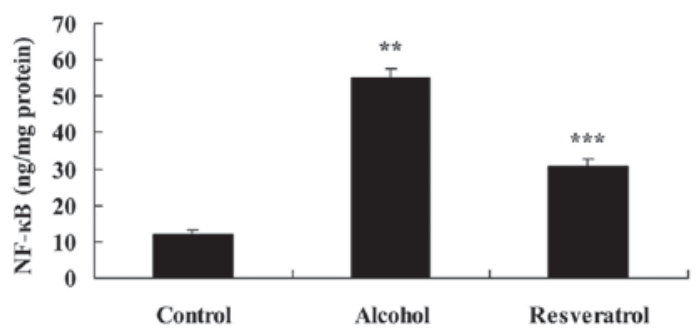

B

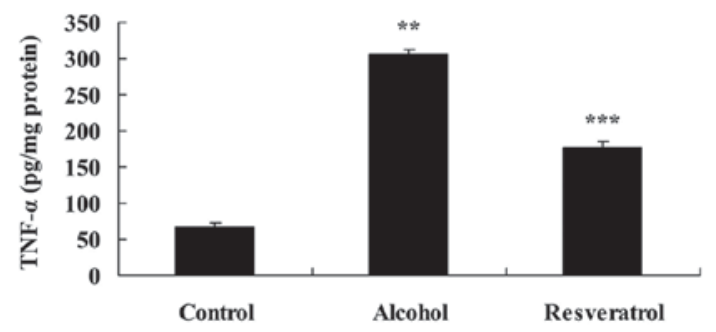

Figure 3. Resveratrol protects against the alcohol-induced inflammatory response. Alcohol-induced levels of (A) NF- $\kappa \mathrm{B}$ and (B) TNF- $\alpha$ (B in alcohol-induced rats. ${ }^{* *} \mathrm{P}<0.01$, compared with the control group; ${ }^{* * * *} \mathrm{P}<0.01$, compared with the alcohol group. NF- $\mathrm{B}$, nuclear factor- $\kappa \mathrm{B}$; TNF- $\alpha$, tumor necrosis factor- $\alpha$.

A

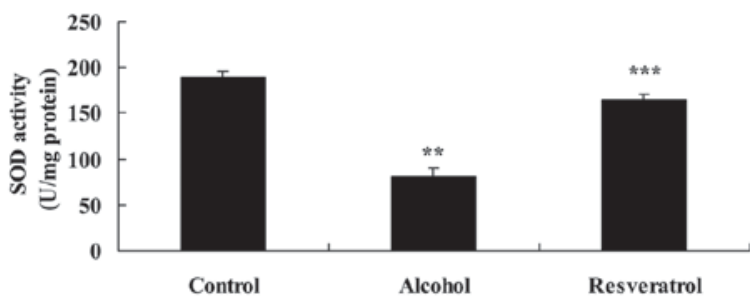

B

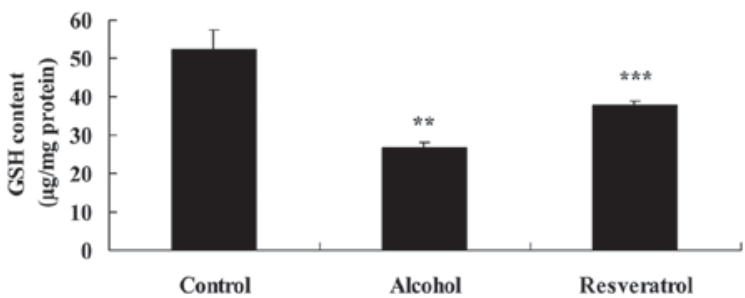

Figure 4. Resveratrol protects alcohol-induced oxidative stress. Alcohol-induced levels of (A) SOD and (B) GSH in alcohol-induced rats. ${ }^{* *} \mathrm{P}<0.01$, compared with the control group; ${ }^{* * * *} \mathrm{P}<0.01$, compared with the alcohol group. SOD, superoxide dismutase; GSH, glutathione.

Statistical analysis. All data are presented as the mean \pm standard error of the mean using SPSS 17.0 (SPSS, Inc., Chicago, IL, USA). Statistical analyses were performed using Student's t-test. $\mathrm{P}<0.05$ was considered to indicate a statistically significant difference. All data show results from at least three independent experiments.

\section{Results}

Resveratrol protects against alcohol-induced neurodegeneration. The chemical structure of resveratrol is shown in Fig. 1. The neuroprotective effects of resveratrol on alcohol-induced neurodegeneration were measured, in which the number of neuron cells were observed in every group. Alcohol inhibited the number of neuron cells in the rats, compared with the number in the control group (Fig. 2). Resveratrol increased the number of neuron cells in the rats, compared with the number in the alcohol-induced model group (Fig. 2).

Resveratrol protects against the alcohol-induced inflammatory response. The present study then examined the effect of resveratrol on the alcohol-induced inflammatory response in rats. The results showed that the expression

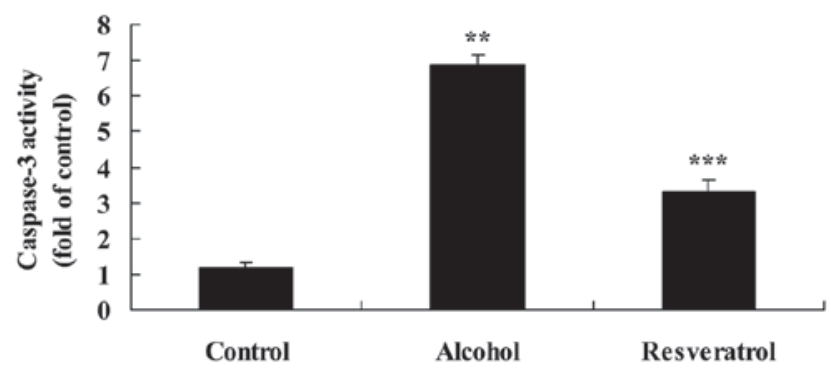

Figure 5. Resveratrol protects against alcohol-induced expression of caspase-3. ${ }^{* *} \mathrm{P}<0.01$, compared with the control group; ${ }^{* * *} \mathrm{P}<0.01$, compared with the alcohol group.

levels of NF- $\kappa \mathrm{B}$ and TNF- $\alpha$ were markedly increased in the alcohol-induced model group, compared with levels in the control group (Fig. 3A and B). Treatment with resveratrol for 4 days reduced the alcohol-induced levels of $N F-\kappa B$ and TNF- $\alpha$ in the alcohol-induced rats (Fig. 3A and B).

Resveratrol protects against alcohol-induced oxidative stress. The present study also examined the expression levels of SOD and GSH using ELISA kits. In the model group, 
A

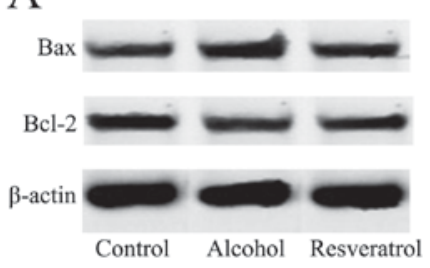

$\mathrm{B}$

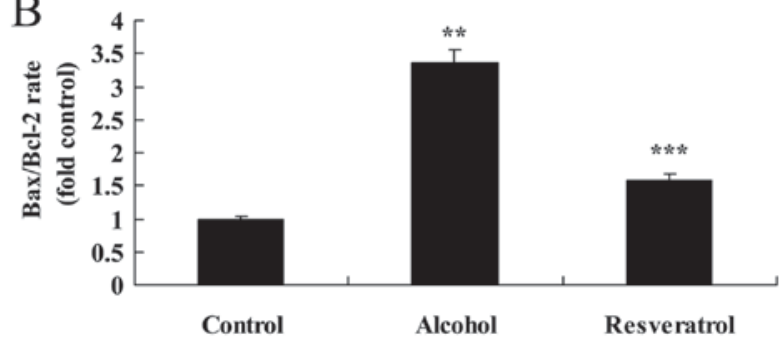

Figure 6. Resveratrol protects against the alcohol-induced expression of Bax/Bcl-2. (A) Protein expression of Bax and Bcl-2 protein using western blot analysis. (B) Statistical analysis of protein expression levels of Bax and Bcl-2. ${ }^{* *} \mathrm{P}<0.01$, compared with the control group; ${ }^{* * * *} \mathrm{P}<0.01$, compared with the alcohol group. Bcl-2, B-cell lymphoma 2; Bax, Bcl-2-associated X protein.
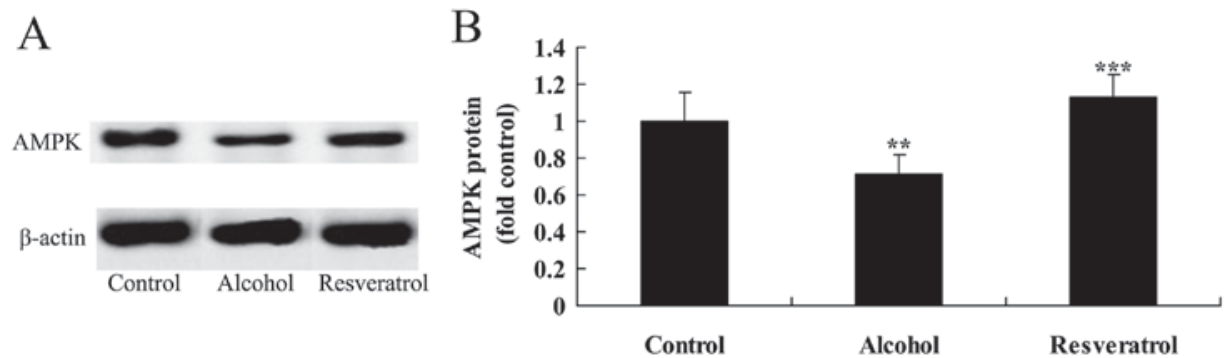

Figure 7. Resveratrol upregulates the expression of AMPK. (A) Protein expression of AMPK using western blot analysis. (B) Statistical analysis of the protein expression of AMPK. ${ }^{* *} \mathrm{P}<0.01$, compared with the control group; ${ }^{* * * *} \mathrm{P}<0.01$, compared with the alcohol group. AMPK, AMP-activated protein kinase.

the expression levels of SOD and GSH were significantly suppressed, compared with the levels in the control group (Fig. 4A and B). Treatment of the alcohol-induced rats with resveratrol significantly increased the expression levels of SOD and GSH in the rats, compared with the number in the model group (Fig. 4A and B).

Resveratrol protects against alcohol-induced caspase-3 activity. The microplate reader analysis of the activity of caspase-3 activity following alcohol exposure revealed that the activity of caspase-3 was increased significantly, compared with that in the control group. However, the activity of caspase-3 was significantly decreased 5 days following treatment with resveratrol (Fig. 5).

Resveratrol protects against alcohol-induced expression of $\mathrm{Bax} / \mathrm{Bcl}$-2. To examine the mechanisms underlying the neuroprotective effects of resveratrol on alcohol-induced $\mathrm{Bax} / \mathrm{Bcl}-2$ ratio in rats, the present study measured the expression of $\mathrm{Bax} / \mathrm{Bcl}-2$ using western blot analysis. Alcohol significantly increased the ratio of $\mathrm{Bax} / \mathrm{Bcl}-2$, compared with that in the control group (Fig. 6). Following the administration of resveratrol, the ratio of $\mathrm{Bax} / \mathrm{Bcl}-2$ was significantly decreased in the rats, compared with that in the model group (Fig. 6).

Resveratrol upregulates the expression of AMPK. The present study also assessed whether the neuroprotective effects of resveratrol affected the expression of AMPK in the alcohol-induced rats. The results of the western blot analysis showed that the protein expression of AMPK was significantly suppressed following alcohol exposure, compared with that in the control group (Fig. 7A and B). By contrast, treatment with resveratrol upregulated the expression of AMPK in the alcohol-induced rats, compared with that in the alcohol-induced model group (Fig. 7A and B).

Resveratrol upregulates the expression of SIRT1. The results of the western blot analysis showed that alcohol inhibited the protein expression of SIRT1 in the rats, compared with that in the control group (Fig. 8A and B). However, pretreatment with resveratrol significantly upregulated the inhibited protein expression of SIRT1 induced by alcohol, compared with the expression in the model group without resveratrol (Fig. 8A and B).

Resveratrol downregulates the expression of $p-p 38$. The results showed that the protein expression of p-p38 was increased following exposure to alcohol, compared with that in the control group (Fig. 9A and B). Treatment with resveratrol significantly decreased the alcohol-induced protein expression of p-p38, compared with the expression in the model group without resveratrol (Fig. 9A and B).

Downregulation of AMPK affects the expression of SIRT1 and $p 38$. In order to provide additional support for the results described above, the present study analyzed the effect of the downregulation of AMPK on the expression of SIRT1 and p38 in the SH-SY5Y cells. The results demonstrated that the downregulation of AMPK suppressed the expression of SIRT1 and activated the expression of p38 in the SH-SY5Y cell model (Fig. 10A-D).

\section{Discussion}

Alcohol has become a commonly consumed beverage. Although a small quantity of alcohol may be beneficial to 

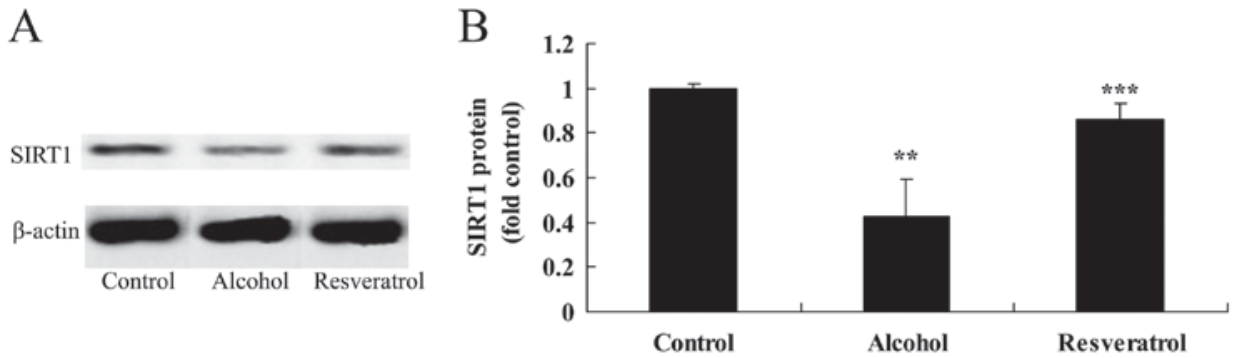

Figure 8. Resveratrol upregulates the expression of SIRT1. (A) Protein expression of SIRT1 using western blot analysis. (B) Statistical analysis of the protein expression of SIRT1. ${ }^{* *} \mathrm{P}<0.01$, compared with the control group; ${ }^{* * *} \mathrm{P}<0.01$, compared with the alcohol group. SIRT1, sirtuin 1.
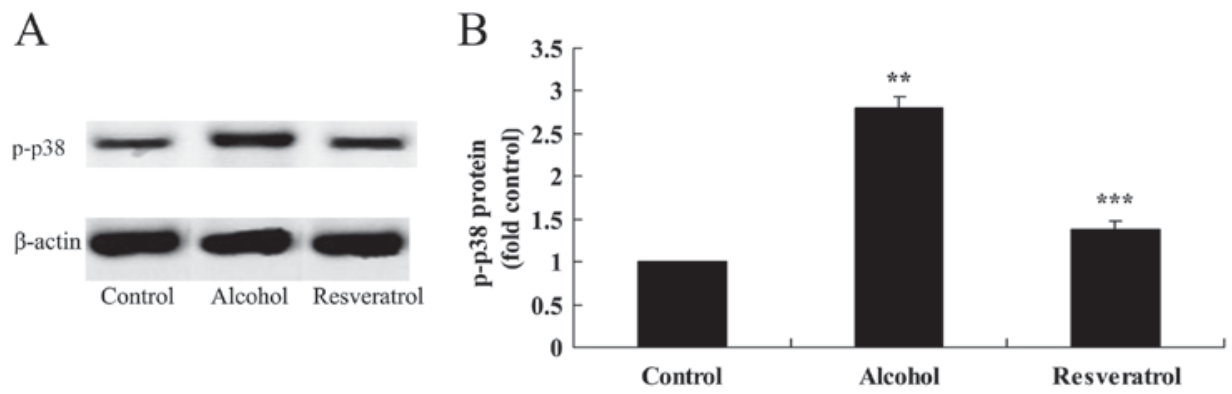

Figure 9. Resveratrol downregulates the expression of p-p38. (A) Protein expression of p-p38 using western blot analysis. (B) Statistical analysis of the protein expression of $\mathrm{p}-\mathrm{p} 38 .{ }^{* *} \mathrm{P}<0.01$, compared with the control group; ${ }^{* * *} \mathrm{P}<0.01$, compared with the alcohol group.

A

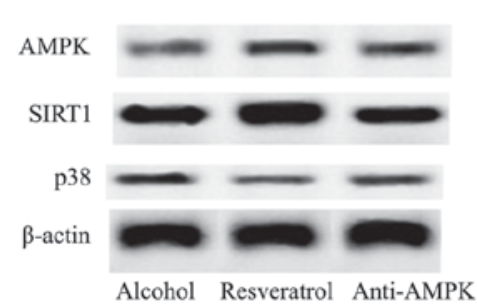

$\mathrm{C}$

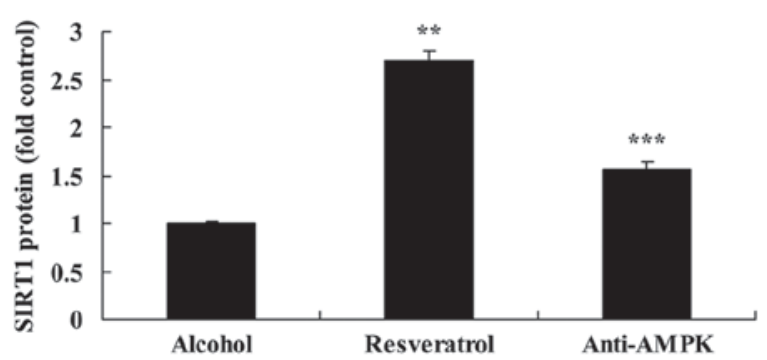

$\mathrm{B}$

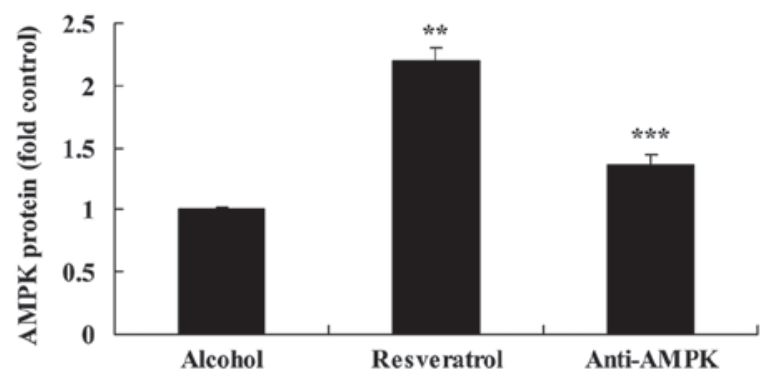

$\mathrm{D}$

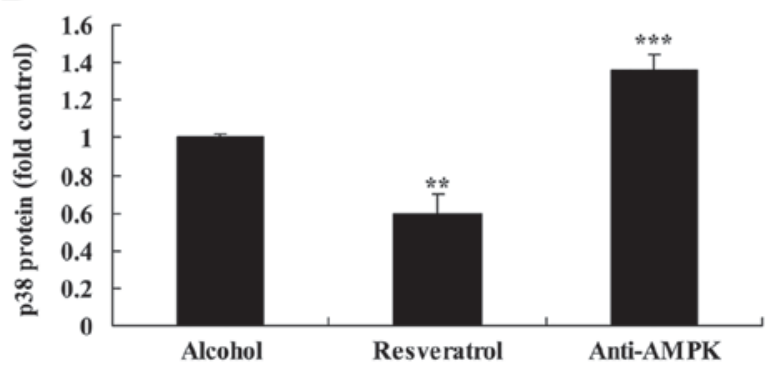

Figure 10. Downregulation of the effect of AMPK on the expression of SIRT1 and p38. (A) Protein expression levels of AMPK, SIRT1 and p38 using western blot analysis. Statistical analysis of protein expression levels of (B) AMPK, (C) SIRT1 and (D) p38. ${ }^{* *} \mathrm{P}<0.01$, compared with the control group; ${ }^{* * * *} \mathrm{P}<0.01$, compared with the alcohol group. AMPK, AMP-activated protein kinase; SIRT1, sirtuin 1.

humans, long-term heavy alcohol intake can cause damage to the digestive system, cardiovascular system, immune system, nervous system and other organs in the body, and can induce cancer $(6,10)$. The potential damage of alcohol to the nervous system is relatively high; it has a direct neurotoxic effect on the central nervous system through the blood brain barrier, induces the apoptosis of nerve cells, reduces the number of synapses, and can lead to organic change in brain function (11). Cognitive impairment is a mild symptom, however, serious consequences include the induction of neurodegenerative diseases, including Alzheimer's disease and Parkinson's disease $(12,13)$. The present study found that resveratrol reduced the number of alcohol-induced microglial cells and neuron cells, and inhibited the increased levels of NF- $\mathrm{kB}$, 
TNF- $\alpha$, SOD and GSH in alcohol-induced rats. Zhang et al reported the neuroprotective effects of resveratrol against glutamate-induced excitotoxicity in neurodegenerative diseases (14), and Abengózar-Vela et al demonstrated that resveratrol reduced inflammatory and oxidative damage in human ocular surface epithelial cells (15).

AMPK has protective and reparative effects on developing and mature neurons, which can enhance the uptake of neurons cultured in vitro and promote the differentiation of neurons (16). Studies have shown that the expression of AMPK is significantly decreased in the substantia nigra of patients (17). In open clinical trials, intraspinal and putamen injections of AMPK have exhibited significant efficacy. The partial protective effect of AMPK protein on glucose-deprived PC12 cells is realized by the direct binding with p38, which partially inhibits p38 nuclear translocation, and inhibits its transcription-dependent pro-apoptotic process; in addition to the binding site of 253-282 bits, sites between may exist (18). The overexpression of AMPK can also inhibit the expression of p38, which can weaken p38-induced pro-apoptosis (19). In the present study, it was found that treatment with resveratrol upregulated the expression of AMPK in the alcohol-induced rats. Guo et al also demonstrated that resveratrol protects against cardiomyocytes through the AMPK-associated pathway (20).

SIRT1 is the most unique homologous protein of SIRT2 in mammals, involved in chromatin remodeling, gene silencing and DNA damage repair processes (21). SIRT1 has a substrate, which is widely used, including transcription factors $\mathrm{p} 53$, forkhead box O and NF-kB (22). The activity of SIRT1 can be regulated by oxidized nicotinamide adenine dinucleotide and nicotinamide and phosphorylation (23). SIRT1 is involved in the regulation of age-related metabolic processes, including fat storage, insulin secretion, glucose metabolism, neuroprotection and apoptosis; therefore, it is possible that Sirtl is associated with the life span of mammals (24). The present study showed that resveratrol significantly upregulated the alcohol-inhibited protein expression of SIRT1 in rats. Sonnemann et al also suggested that resveratrol significantly promotes the protein expression of SRT1 in SK-N-MC cells (9).

Tumor suppressor p38 can inhibit cell proliferation through cell cycle arrest, apoptosis and the response to cell aging induced by stress from different cells (25). There is data showing that tumors without mutant p38 are likely to have other defects in the p38 signaling pathway, which has an important effect on the form of cancer (26). In addition, experiments have shown that p38 is a non-histone target gene, which is deacetylated by Sirtl and the first to have been found (27). The present found that treatment with resveratrol significantly reduced the alcohol-induced protein expression of p-p38. Wu et al (28) reported that resveratrol induces the apoptosis of human chronic myelogenous leukemia cells through p38. Of note, the downregulation of AMPK suppressed the expression of SIRT1 and activation of p38 in the SH-SY5Y cell model.

In conclusion, the data obtained in the present study showed that resveratrol protected against alcohol-induced neurodegeneration. The results also provided novel mechanistic insights by which SH-SY5Y cells are important in mediating AMPK/SIRT1/p38 signaling.

\section{Acknowledgements}

The present study was supported by the National Natural Science Foundation of China (grant no. 81673727).

\section{References}

1. Goodlett CR and Eilers AT: Alcohol-induced Purkinje cell loss with a single binge exposure in neonatal rats: A stereological study of temporal windows of vulnerability. Alcohol Clin Exp Res 21: 738-744, 1997.

2. Qin L and Crews FT: NADPH oxidase and reactive oxygen species contribute to alcohol-induced microglial activation and neurodegeneration. J Neuroinflammation 9: 5, 2012.

3. Takeuchi M and Saito T: Cytotoxicity of acetaldehyde-derived advanced glycation end-products (AA-AGE) in alcoholic-induced neuronal degeneration. Alcohol Clin Exp Res 29 (12 Suppl): 220S-224S, 2005.

4. Weiss B: The first 83 and the next 83: Perspectives on neurotoxicology. Neurotoxicology 30: 832-850, 2009.

5. Myers J, London L and Lucchini RG: Neurotoxicology and development: Human, environmental and social impacts. Neurotoxicology 45: 217-219, 2014.

6. Berman JW, Carson MJ, Chang L, Cox BM, Fox HS, Gonzalez RG, Hanson GR, Hauser KF, Ho WZ, Hong JS, et al: NeuroAIDS, drug abuse, and inflammation: Building collaborative research activities. J Neuroimmune Pharmacol 1: 351-399, 2006.

7. Trickler WJ, Lantz SM, Murdock RC, Schrand AM, Robinson BL, Newport GD, Schlager JJ, Oldenburg SJ, Paule MG, Slikker W Jr, et al: Silver nanoparticle induced blood-brain barrier inflammation and increased permeability in primary rat brain microvessel endothelial cells. Toxicol Sci 118: $160-170,2010$.

8. Park I, Lee Y, Kim HD and Kim K: Effect of resveratrol, a SIRT1 activator, on the interactions of the CLOCK/BMAL1 complex. Endocrinol Metab (Seoul) 29: 379-387, 2014

9. Sonnemann J, Kahl M, Siranjeevi PM, Blumrich A, Blümel L, Becker S, Wittig S, Winkler R, Krämer OH and Beck JF: Reverse chemomodulatory effects of the SIRT1 activators resveratrol and SRT1720 in Ewing's sarcoma cells: Resveratrol suppresses and SRT1720 enhances etoposide- and vincristine-induced anticancer activity. J Cancer Res Clin Oncol 142: 17-26, 2016.

10. Allen JW, Mutkus LA and Aschner M: Chronic ethanol produces increased taurine transport and efflux in cultured astrocytes. Neurotoxicology 23: 693-700, 2002.

11. Wan W and DePetrillo PB: Ritonavir protects hippocampal neurons against oxidative stress-induced apoptosis. Neurotoxicology 23: 301-306, 2002.

12. Overstreet DH: Organophosphate pesticides, cholinergic function and cognitive performance in advanced age. Neurotoxicology 21 : 75-81, 2000.

13. Polizzi S, Pira E, Ferrara M, Bugiani M, Papaleo A, Albera R and Palmi S: Neurotoxic effects of aluminium among foundry workers and Alzheimer's disease. Neurotoxicology 23: 761-774, 2002.

14. Zhang LN, Hao L, Wang HY, Su HN, Sun YJ, Yang XY, Che B, Xue J and Gao ZB: Neuroprotective effect of resveratrol against glutamate-induced excitotoxicity. Adv Clin Exp Med 24: 161-165, 2015.

15. Abengózar-Vela A, Calonge M, Stern ME, González-Garcia MJ and Enriquez-De-Salamanca A: Quercetin and resveratrol decrease the inflammatory and oxidative responses in human ocular surface epithelial cells. Invest Ophthalmol Vis Sci 56: 2709-2719, 2015.

16. Leyton L, Hott M, Acuña F, Caroca J, Nuñez M, Martin C, Zambrano A, Concha MI and Otth C: Nutraceutical activators of AMPK/Sirt1 axis inhibit viral production and protect neurons from neurodegenerative events triggered during HSV-1 infection. Virus Res 205: 63-72, 2015.

17. Choi JS, Park C and Jeong JW: AMP-activated protein kinase is activated in Parkinson's disease models mediated by 1-methyl-4-phenyl-1,2,3,6-tetrahydropyridine. Biochem Biophys Res Commun 391: 147-151, 2010.

18. Hao W, Takano T, Guillemette J, Papillon J, Ren G and Cybulsky AV: Induction of apoptosis by the Ste20-like kinase SLK, a germinal center kinase that activates apoptosis signal-regulating kinase and p38. J Biol Chem 281: 3075-3084, 2006. 
19. Liu K, Yang Y and Mansbridge J: Comparison of the stress response to cryopreservation in monolayer and three-dimensional human fibroblast cultures: Stress proteins, MAP kinases, and growth factor gene expression. Tissue Eng 6: 539-554, 2000

20. Guo S, Yao Q, Ke Z, Chen H, Wu J and Liu C: Resveratrol attenuates high glucose-induced oxidative stress and cardiomyocyte apoptosis through AMPK. Mol Cell Endocrinol 412: 85-94, 2015.

21. Yoshida K, Bacal J, Desmarais D, Padioleau I, Tsaponina O, Chabes A, Pantesco V, Dubois E, Parrinello $\mathrm{H}$, Skrzypczak M, et al: The histone deacetylases sir2 and rpd3 act on ribosomal DNA to control the replication program in budding yeast. Mol Cell 54: 691-697, 2014.

22. Zill OA, Scannell D, Teytelman L and Rine J: Co-evolution of transcriptional silencing proteins and the DNA elements specifying their assembly. PLoS Biol 8: e1000550, 2010.

23. Gallagher JE, Babiarz JE, Teytelman L, Wolfe KH and Rine J: Elaboration, diversification and regulation of the Sir1 family of silencing proteins in Saccharomyces. Genetics 181: 1477-1491, 2009.
24. Hou Z, Bernstein DA, Fox CA and Keck JL: Structural basis of the Sirl-origin recognition complex interaction in transcriptional silencing. Proc Natl Acad Sci USA 102: 8489-8494, 2005.

25. Donauer J, Schreck I, Liebel U and Weiss C: Role and interaction of p53, BAX and the stress-activated protein kinases p38 and JNK in benzo(a)pyrene-diolepoxide induced apoptosis in human colon carcinoma cells. Arch Toxicol 86: 329-337, 2012.

26. Dziegielewska B, Brautigan DL, Larner JM and Dziegielewski J: T-type $\mathrm{Ca} 2+$ channel inhibition induces p53-dependent cell growth arrest and apoptosis through activation of p38-MAPK in colon cancer cells. Mol Cancer Res 12: 348-358, 2014.

27. Hishida T, Nozaki Y, Nakachi Y, Mizuno Y, Iseki H, Katano M, Kamon M, Hirasaki M, Nishimoto M, Okazaki Y and Okuda A: Sirt1, p53, and p38(MAPK) are crucial regulators of detrimental phenotypes of embryonic stem cells with Max expression ablation. Stem Cells 30: 1634-1644, 2012.

28. Wu XP, Xiong M, Xu CS, Duan LN, Dong YQ, Luo Y, Niu TH and Lu CR: Resveratrol induces apoptosis of human chronic myelogenous leukemia cells in vitro through p38 and JNK-regulated H2AX phosphorylation. Acta Pharmacol Sin 36: 353-361, 2015. 